\title{
ARIADNE: A novel photographic 1-ton dual-phase LArTPC
}

Konstantinos Mavrokoridis*, D. Hollywood, K. Majumdar, K. J. McCormick, B. Philippou, S. Powell, A. Roberts, N. A. Smith, G. Stavrakis, C. Touramanis, J. Vann

University of Liverpool

Department of Physics, Oliver Lodge Bld, Oxford Street, Liverpool, L69 7ZE, UK

E-mail:k.mavrokoridiseliverpool.ac.uk

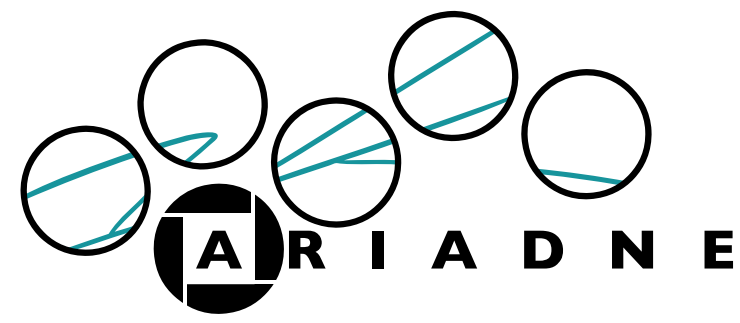

\begin{abstract}
ARIADNE is a 1-ton dual-phase Time Projection Chamber (TPC) with a novel optical readout. The secondary scintillation light produced in the holes of a THick Gas Electron Multiplier (THGEM) is captured by Electron Multiplying Charge Coupled Device (EMCCD) cameras or fast TPX3Cams mounted externally via glass viewports. Optical readout has the potential to be a cost effective alternative to charge readout in future LArTPCs. First ever optical images of argon interactions from the operation of the ARIADNE detector at the CERN T9 testbeam are presented. 3D track reconstruction of cosmic muons collected at the University of Liverpool is also detailed.
\end{abstract}

ARIADNE is an ERC funded experiment, grant number 677927

The 21st international workshop on neutrinos from accelerators (NuFact2019)

August 26 - August 31, 2019

Daegu, Korea

\footnotetext{
* Speaker.
} 


\section{Introduction}

Kiloton-scale Liquid Argon Time Projection Chambers (LArTPCs) for neutrino physics are soon expected to be realised. The DUNE project [1] is proposing the use of four 17,000 ton LArTPCs. The cost and complexity of these large scale detectors means that early R\&D work has the potential for a large return on investment. ARIADNE (ARgon ImAging DetectioN chambEr) is a 1-ton dual-phase LArTPC, built to pursue the initial demonstration of optical readout $[2,3]$ on a larger scale. Optical readout presents many advantages over the traditional charge readout. In this paper a summary of results from operation at CERN's T9 beamline as well as fast imaging of LAr interactions with a TP3Cam are detailed.

\section{The ARIADNE Detector and Operation Principle}

Figure 1 shows a general overview of the ARIADNE detector and the operation principle. The ARIADNE TPC is $54 \times 54 \mathrm{~cm}(x, y)$ with a total drift length of $80 \mathrm{~cm}(z)$. Four PMTs are installed below the TPC, providing detector triggering via the detection of primary scintillation (S1) light, and also performing auxiliary detection of secondary scintillation (S2) light. The field cage of the TPC is composed of a total of 79 field-shaping rings, spaced $1 \mathrm{~cm}$ apart. The cathode is installed at the base of the field cage and can be biased up to $80 \mathrm{kV}$, providing a drift field of up to $1 \mathrm{kV} / \mathrm{cm}$ within the TPC. Above the field cage is an extraction grid, with a Thick Gas Electron Multiplier (THGEM) $11 \mathrm{~mm}$ above the grid. The space between the extraction grid and THGEM forms the extraction region, which is nominally biased with an extraction field of $3 \mathrm{kV} / \mathrm{cm}$. To shift the $128 \mathrm{~nm}$ Vacuum Ultra Violet (VUV) light that is produced in the THGEM holes to $420 \mathrm{~nm}$, a glass plate placed $2 \mathrm{~mm}$ above the THGEM plane is coated with Tetraphenyl Butadiene (TPB). Four EMCCD cameras (or TPX3Cam), installed outside of the cryostat, look down at the THGEM through optical viewports and capture the S2 light produced in the THGEM holes. A more detailed description of the detector design and operation can be found in [4].

\section{ARIADNE at the CERN T9 Beamline}

The ARIADNE detector was operated at the T9 beamline at CERN's East Hall [5] for 18 days during March and April of 2018, and data from electrons, muons, pions and protons (as well as their anti-particles) were collected at momenta between 0.5 and $8.0 \mathrm{GeV} / \mathrm{c}$. A total of just under 800,000 events were collected, split evenly between the positive and negative polarities. Information concerning the relative fractions of each particle species, as measured at the T9 source, can be found in [5]. Figure 2 displays a selection of raw data images of negative polarity particle tracks taken on the CERN T9 beamline by the ARIADNE detector. This represents the first optical imaging of LAr-particle interactions on a beamline, using a dual-phase LArTPC. The THGEM field for these results was $27 \mathrm{kV} / \mathrm{cm}$. At this field the light produced is within the proportional electroluminescence regime. The detector configuration and more results taken during beamline operation are detailed in [4]. 

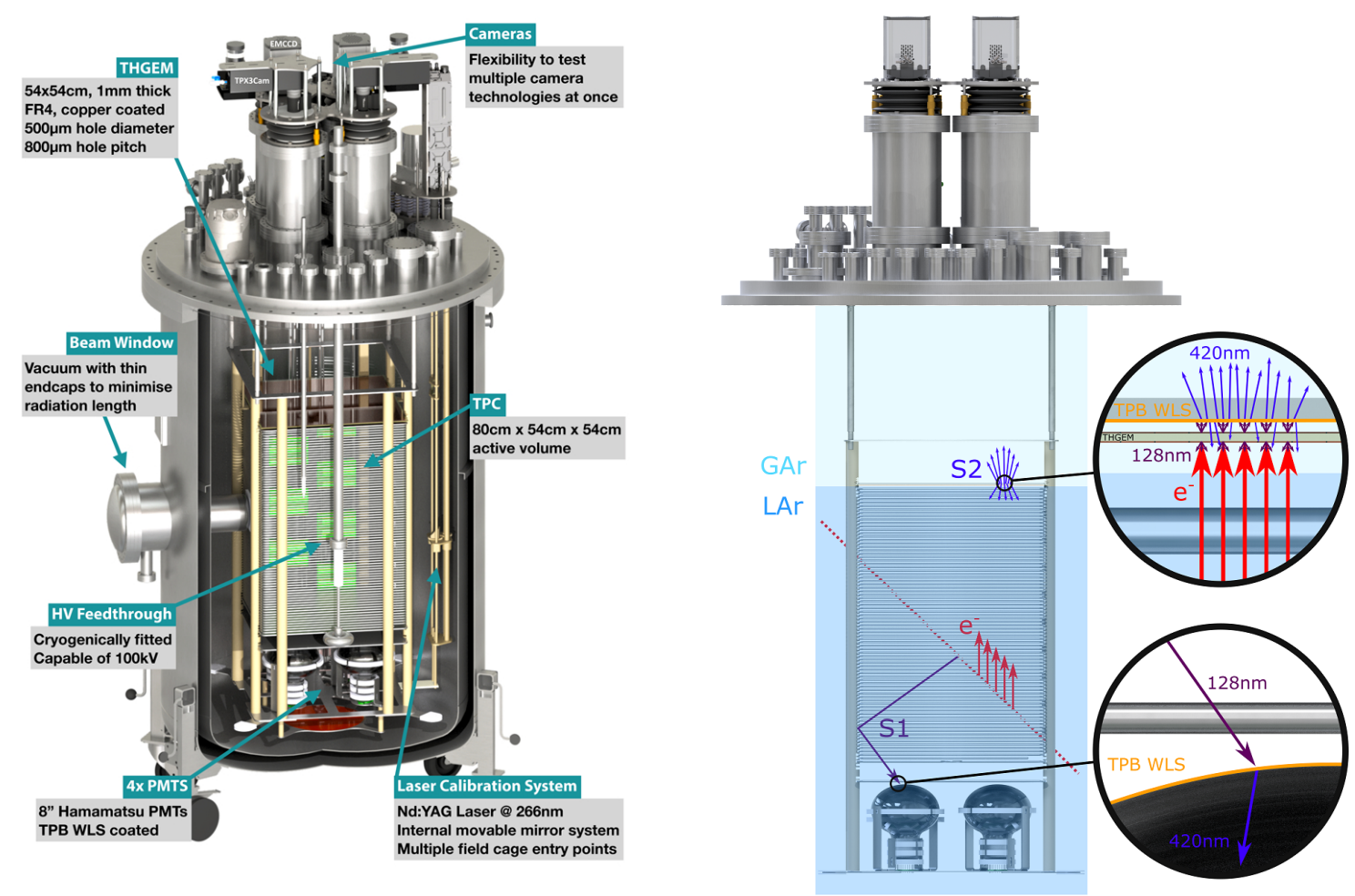

Figure 1: (Left) The ARIADNE detector. (Right) The Operation Principle. A charged particle enters the active region of the TPC and interacts with the liquid phase, producing an S1 signal, seen by the PMTs, and ionising the argon. The free electrons drift upwards in response to an electric field and are extracted into the gas phase. These electrons are further accelerated by the THGEM producing an S2 signal. This is imaged by the cameras.

\section{Fast Imaging with TPX3Cam}

To overcome the speed limitation of the EMCCDs, a new camera based on TPX3 technology was tested. The first successful demonstration of this technology was performed using the ARIADNE prototype vessel in low pressure $\mathrm{CF}_{4}$ by the authors [6]. Following this demonstration, one EMMCD camera from ARIADNE was replaced with a TPX3Cam. The operation principle of the TPX3Cam is shown in Figure 3. The assembly consists of a lens coupled to a Photonis Cricket image intensifier $(\approx 33 \%$ QE at $420 \mathrm{~nm}$ ), which boosts the $\mathrm{S} 2$ light signal. The incident light is converted to charge through a photo-cathode, and these electrons are then multiplied through a dual microchannel plate (MCP). The amplified charge is then converted back to light using a phosphor screen. The photons from the phosphor screen are focused to light sensitive silicon, bump bonded to a TPX3 chip ( $55 \mu \mathrm{m}, 256 \times 256$ pixel array). Light incident onto the silicon will excite an electron-hole pair. The TPX3 ASIC then accumulates these electrons. If the overall charge exceeds a user defined threshold (a typical minimum operating threshold is around $500 \mathrm{e}^{-}$), the TPX3 chip registers a hit (with $1.6 \mathrm{~ns}$ resolution). This is known as the Time of Arrival (ToA). The accumulated charge is then discharged at a continuous rate, through the capacitor, until the charge has dropped below the predefined threshold. The length of time for which the charge remains over threshold is known as Time over Threshold (ToT). This value is directly proportional to the initial 


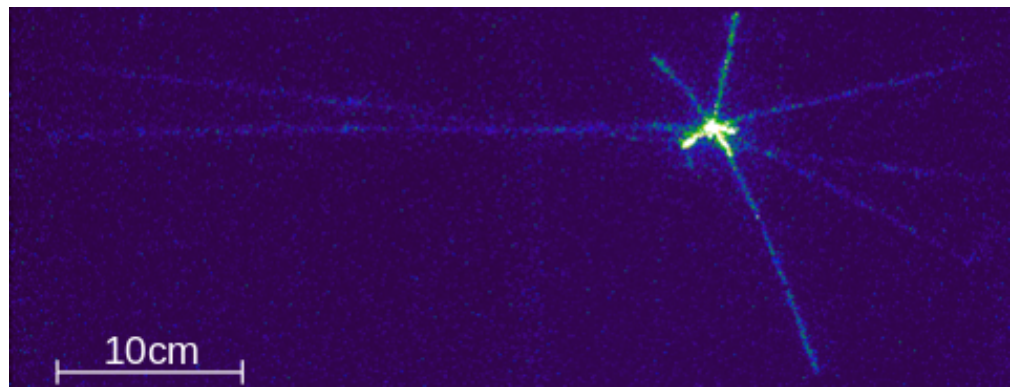

3.0 GeV/c anti-proton candidate, maximum intensity $=21894$ ADU

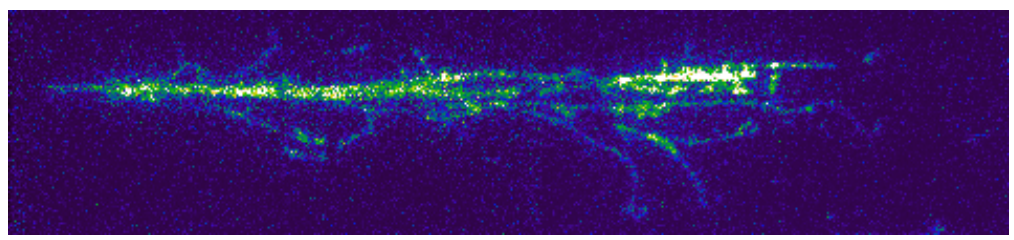

$5.0 \mathrm{GeV} / \mathrm{c}$ electromagnetic shower, maximum intensity $=12916 \mathrm{ADU}$

Figure 2: Beamline events as captured by the ARIADNE detector

incident photon flux on the silicon sensor (similar to intensity in an EMCCD). ToT is recorded with 10-bit resolution. The TPX 3 chip then sends a packet containing 4 pieces of information - $x, y$ pixel position, ToA $(z)$ and ToT. This allows for full 3D reconstruction using a single device. The TPX3 camera produces these information packets in a data driven format (i.e. records pixels only above the user defined threshold). The maximum readout rate is $80 \mathrm{Mhits} / \mathrm{s}$. These properties make TPX3 ideal for optical readout, due to the high readout rate, natively 3D raw data, with low storage due to zero suppression.

Figure 4 shows cosmic muons LAr interactions taken with a single TPX3Cam mounted on the ARIADNE detector. The field in the THGEM holes was $29 \mathrm{kV} / \mathrm{cm}$ and the spatial resolution in this configuration is $1.1 \mathrm{~mm} /$ pixel (similar to the EMCCDs in 4 bin mode). The left hand image shows $1.75 \mathrm{~s}$ of continuous streaming. This corresponds to a $2.9 \mathrm{~km}$ drift (the drift velocity in LAr is $0.16 \mathrm{~cm} / \mu \mathrm{m}$ at $0.5 \mathrm{kV} / \mathrm{cm}$ ). The right hand side images show a $\approx 50 \mu$ s drift snapshot, corresponding to $\approx 10 \mathrm{~cm}$ cosmic muon tracks (with delta ray).
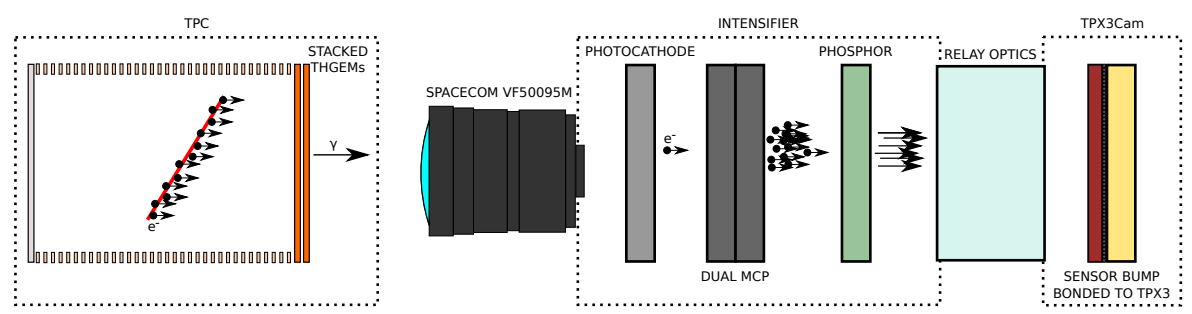

Figure 3: The operation principle of a TPXCam. 

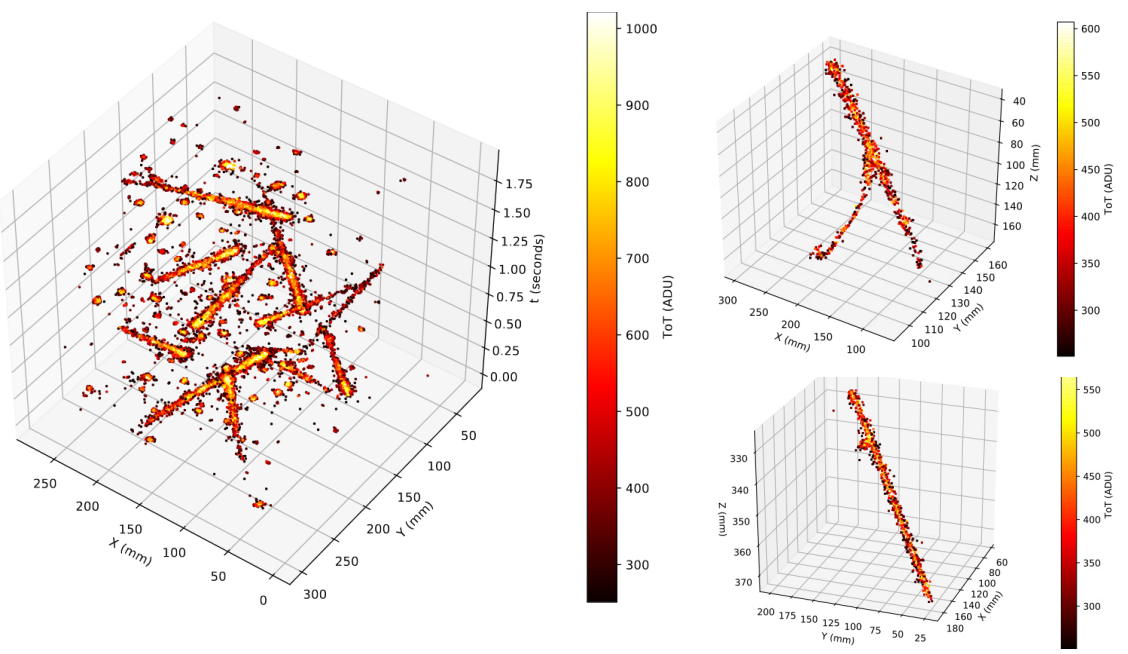

Figure 4: Cosmic muon LAr interactions taken with a TPX3Cam on ARIADNE. The left image represents $1.75 \mathrm{~s}$ continuous streaming. The right tracks are $\approx 12 \mathrm{~cm}$ (top) and $\approx 5 \mathrm{~cm}$ (bottom) long, respectively.

\section{Outlook}

Optical readout shows great promise in terms of performance and cost-efficiency for kiloton scale dual-phase LAr detectors and could conceivably be used for the fourth DUNE module - the technology for which has not yet been decided. Key benefits using a TPX3cam system include: (1) raw data is natively $3 \mathrm{D},(2)$ very fast readout rates (80 MHits/s), (3) zero suppressed readout, (4) well established physics sensor, (5) low cost, (6) same readout possible for dual-phase or gas TPCs. In the imminent future the TPX4 sensor is expected to be released which will have a larger size with $448 \times 512$ pixel array. Considering the $720 \mathrm{~m}^{2}$ area of the proposed DUNE module, the entire detector could be read out with 320 TPX4 cameras, each looking at a field of view of $1.5 \times 1.5 \mathrm{~m}$ with $\approx 3 \mathrm{~mm} /$ pixel resolution.

\section{References}

[1] The DUNE Collaboration, Long-Baseline Neutrino Facility (LBNF) and Deep Underground Neutrino Experiment (DUNE) Conceptual Design Report Volume 1: The LBNF and DUNE Projects, arXiv:1601.05471

[2] K. Mavrokoridis et al., Optical Readout of a Two Phase Liquid Argon TPC using CCD Camera and THGEMs, Journal of Instrumentation, Volume 9, Issue 2 (2014), Page P02006

[3] K. Mavrokoridis et al., First Demonstration of Imaging Cosmic Muons in a Two-Phase Liquid Argon TPC using an EMCCD Camera and a THGEM, JINST, Vol. 10, Issue 10 (2015), P10004

[4] D. Hollywood et al., ARIADNE - A Novel Optical LArTPC: Technical Design Report and Initial Characterisation using the CERN T9 Testbeam and Cosmic Muons, arXiv:1910.03406

[5] CERN - East Area Documentation, http://sba.web.cern.ch/sba/BeamsAndAreas/East/East.htm

[6] A. Roberts et al., First demonstration of 3D optical readout of a TPC using a single photon sensitive Timepix3 based camera, JINST, Vol. 14, Issue 6 (2019), P06001 